\title{
Enterocolitis Infectious, CTCAE
}

National Cancer Institute

\section{Source}

National Cancer Institute. Enterocolitis Infectious, CT CAE. NCI Thesaurus. Code

C143444.

A disorder characterized by an infectious process involving the small and large intestines. 